\title{
High-fat treatment prevents postoperative cognitive dysfunction in a hyperlipidemia model by protecting the blood-brain barrier via Mfsd2a-related signaling
}

\author{
XIAO-PING ZHANG ${ }^{1}$, YU-RU LIU ${ }^{2}$, MEI CHAI $^{1}$, HAI-TAO YANG ${ }^{1}$, GUAN WANG $^{1}$, MEI HAN $^{1}$ and DONG-BAI LI ${ }^{1}$ \\ ${ }^{1}$ Department of Anesthesiology, The Second Affiliated Hospital of Dalian Medical University, Dalian, Liaoning 116011; \\ ${ }^{2}$ Department of Anesthesiology, Xilingol League Hospital, Inner Mongolia Autonomous Region,
}

Chifeng, Xilinhot 026000, P.R. China

Received February 23, 2019; Accepted August 12, 2019

DOI: $10.3892 / \mathrm{mmr} .2019 .10675$

\begin{abstract}
Damage to the blood-brain barrier (BBB) resulting from systemic inflammation caused by surgical trauma is associated with cognitive dysfunction, and individuals with hyperlipidemia are more sensitive to such impairment. The present study was designed to ascertain whether dexmedetomidine (Dex) treatment could reduce the incidence of cognitive dysfunction following surgery in a hyperlipidemia model. Hyperlipidemia was induced in Sprague-Dawley rats (male, 6-7 months old) by consuming a high-fat diet, and rats were divided into three groups $(\mathrm{n}=10$ each) and underwent: exploratory laparotomy to introduce surgical trauma (surgery group), laparotomy and Dex treatment (surgery+Dex group), or sham surgery (sham group). Learning, memory and exploration behavior were assessed using the Morris water maze. Concentrations of tumor necrosis factor (TNF)- $\alpha$ and interleukin (IL)-1 $\beta$, were determined by enzyme-linked immunosorbent assay. BBB permeability was assessed by Evans blue staining. Relative major facilitator superfamily domain-containing protein 2 (Mfsd2a) mRNA expression was
\end{abstract}

Correspondence to: Dr Dong-Bai Li, Department of Anesthesiology, The Second Affiliated Hospital of Dalian Medical University, 467 Zhongshan Road, Shahekou, Dalian, Liaoning 116011, P.R. China E-mail: dblee69@hotmail.com

Abbreviations: $\mathrm{BBB}$, blood-brain barrier; $\mathrm{POCD}$, postoperative cognitive dysfunction; CNS, central nervous system; Mfsd2a, Major facilitator superfamily domain-containing protein 2 ; TNF- $\alpha$, tumor necrosis factor- $\alpha$; IL-1 $\beta$, interleukin-1 $\beta$; TRPM2, transient receptor potential cation channel, subfamily $\mathrm{M}$, member 2; TRPV1, transient receptor potential cation channel, subfamily $\mathrm{V}$, member 1 ; TGs, triglycerides; TC, total cholesterol; MWM, Morris water maze; OD, optical density; qPCR, quantitative real-time PCR; $A \beta, \beta$-amyloid protein; HDL, high-density lipoprotein; VLDL, very low-density lipoprotein; LDL, low-density lipoprotein; BDNF, brain-derived neurotrophic factor; LTP, long-term potentiation

Key words: postoperative cognitive dysfunction, blood-brain barrier, Mfsd2a, dexmedetomidine, hyperlipidemia, TNF- $\alpha$, IL-1 $\beta$ determined by quantitative PCR. In the Morris water maze test, the time and distance ratio for the surgery group was significantly lower than those of the sham and surgery+Dex groups $(\mathrm{P}<0.05)$. In addition, the TNF- $\alpha$ concentrations in the sham and surgery+Dex groups were lower than that in the surgery group $(\mathrm{P}<0.05$ on days 1 and 3$)$. Evans Blue staining was increased in the surgery group on day $1(\mathrm{P}<0.01)$. Mfsd2a mRNA expression was higher in the sham and surgery+Dex groups compared with that noted in the surgery group $(\mathrm{P}<0.05)$. In conclusion, Dex treatment decreased the incidence of cognitive dysfunction following surgical trauma in a hyperlipidemia rat model. We demonstrated that Dex stabilized BBB integrity through increased $M f s d 2 a$ gene expression.

\section{Introduction}

Postoperative cognitive dysfunction (POCD) is a central nervous system (CNS) complication that can occur at any age but is higher among elderly patients who are exposed to major surgery under general anesthesia (1). With the continuous aging of the population, the prevalence and consequences of POCD are increasing, and interventions that prevent POCD represent an unmet medical need. Across a variety of studies, POCD has been consistently associated with prolonged hospitalization, reduced quality of life, and increased morbidity (2). Factors that can increase the risk of POCD include increasing age, severity of the surgery, duration of anesthesia, occurrence of complications, pre-existing cognitive impairment, hemorrhagic shock, and education level $(3,4)$. Hyperlipidemia is among the many risk factors associated with the development of POCD $(3,4)$. The development and maintenance of cerebral function relies on circulating lipids. Abnormalities in gene expression, function, and regulation induce changes in lipid metabolism that predispose patients to hyperlipidemia, which has been shown to be associated with the development and progression of Alzheimer's disease $(5,6)$. The introduction of hyperlipidemia into POCD-related research better reflects the clinical scenario where increased lipids correlate with the incidence of POCD.

The blood-brain barrier (BBB) provides a protective barrier between plasma and neuronal cells. While the selective 
permeability of the BBB has important biological significance for maintaining the normal physiological state of the CNS, it also provides a barrier preventing passage of drugs into the CNS (7). The BBB has been shown to be disturbed in a variety of diseases, and animal models of these conditions have been shown to display cognitive impairments leading to Alzheimer's disease (8). The permeability of the BBB can also increase in response to inflammation, leading to damage to neuronal function.

Major facilitator superfamily domain-containing protein 2 (Mfsd2a) is exclusively expressed in endothelial cells of the $\mathrm{BBB}$ and is regulated by pericytes to facilitate $\mathrm{BBB}$ integrity (9). $M f s d 2 a$-deficient mice exhibit neurological abnormalities, including ataxia (10). Tumor necrosis factor (TNF)- $\alpha$ is an important mediator in the initiation and amplification of the inflammatory cascade. Activation of immune cells in the peripheral circulation in response to inflammation can result in learning and memory dysfunction in animals (11). Interleukin (IL)-1 $\beta$ is also involved in the inflammatory response, and upregulated expression of IL-1 $\beta$ in the brain can lead to cognitive impairment (12).

In recent years, the protective effects of various anesthetics have been observed and studied. Dexmedetomidine (Dex), a selective agonist of the $\alpha 2$-adrenergic receptor, is widely used in intensive care units due to its sedative and analgesic effects. A large body of recent work supports its favorable effects for improving outcomes and long-term brain function in the critically ill. The source of these benefits may lie in the neuroprotective properties that are seen in experimental models and in the clinical setting, in which it can attenuate delirium, preserve sleep architecture, preserve ventilatory drive and decrease sympathetic tone and inflammatory response (13-15). In a rat model of focal cerebral ischemia, Dex decreased the total ischemic volume by $40 \%$ in the cortex compared to control treatment with $\mathrm{NaCl}$, and a study examining whether DEX can ameliorate long-term cognitive dysfunction indicated that pre-treatment with DEX may improve long-term cognitive function and protect against neuronal degeneration (16). Indeed, Dex treatment was found to reduce cerebral ischemia-induced oxidative stress, apoptosis, and intracellular $\mathrm{Ca}^{2+}$ signaling by inhibiting transient receptor potential cation channel subfamily M member 2 (TRPM2) and transient receptor potential cation channel subfamily $\mathrm{V}$ member 1 (TRPV1) in the rat hippocampus and dorsal root ganglion (17).

From the research observations described above, we hypothesized that the incidence of POCD will be higher in rats with hyperlipidemia than in controls and that Dex treatment may protect against cognitive impairment through actions on Mfsd2a.

\section{Materials and methods}

Animals. Healthy male Sprague-Dawley rats (6-7 months old, weighing 200-250 g) were purchased from the Dalian Medical University Laboratory Animal Center and individually housed in $24 \times 24 \times 36 \mathrm{~cm}$ cages in a room with a temperature of $20 \pm 2^{\circ} \mathrm{C}$ and humidity of $50 \pm 10 \%$. Rats had access to sufficient food and water and were kept on a 12:12 light/dark cycle (lights on at 9:00 a.m.). All rats were allowed to adapt to their new environment for 7 days prior to experiments. Any rats with poor eyesight or swimming ability, as judged by the inability to swim to the platform shown to them immediately prior to their placement in the water, would have been excluded, but none of the rats obtained for this experiment met these criteria. All animal procedures were approved by the Animal Experiment Ethics Committee of Dalian Medical University, China. All studies involving animals are carried out in accordance with the ARRIVE guidelines for reporting experiments involving animals.

\section{Hyperlipidemia model}

High-fat emulsion production. The composition of the high-fat emulsion was chosen based on previous research (18). A $200-\mathrm{ml}$ beaker was put into a $60^{\circ} \mathrm{C}$ water bath, and $30 \mathrm{ml}$ distilled water, $20 \mathrm{ml}$ propylene glycol, and $2 \mathrm{~g}$ sodium deoxycholate were added and fully dissolved. In a separate beaker, $25 \mathrm{~g}$ fat, $10 \mathrm{~g}$ cholesterol, $1 \mathrm{~g}$ propylthiouracil, and $25 \mathrm{ml}$ Tween-80 were added, and the beaker was heated to $100^{\circ} \mathrm{C}$. The contents of the two beakers were combined by stirring the contents of the first beaker into that of the second beaker. The mixture was stored at $4^{\circ} \mathrm{C}$ and melted in a water bath at $37^{\circ} \mathrm{C}$ prior to use.

Generation of the animal model. Two initial groups of rats were developed: a normal diet-fed group and high-fat-treated group (Fig. 1). Every day between 8:00 and 9:00 am, rats in the high-fat-treated group $(n=30)$ were fed the high-fat emulsion preparation by gavage at a dosage of $10 \mathrm{ml} / \mathrm{kg}$ body weight. The rats were fed this diet for 14 days to induce hyperlipidemia. Rats in the normal diet-fed group $(n=30)$ were administered the same doses of saline.

Following the last gavage treatment, rats were fasted for $8 \mathrm{~h}$. Then 2-3 ml blood was collected in heparin anticoagulation tubes from the orbital venous plexus, and plasma was collected via centrifugation $(1,000 \mathrm{x} \mathrm{g}$ for $10 \mathrm{~min})$. Cholesterol was measured using a total cholesterol (TC) enzyme-linked immunosorbent assay (ELISA) kit (American TSZ Co.). Triglyceride (TG) levels were measured using a glycerol three-lipid kit (F001-2 GPO-PAP; Nanjing Institute of Biological Engineering). All assays were performed according to the manufacturer's instructions. After successful establishment of the hyperlipidemia model, navigation training was performed for 6 days. During the training period, the high-fat-treated group rats continued to receive gavage at the dose of $10 \mathrm{mg} / \mathrm{kg}$ to maintain elevated cholesterol levels in the plasma. Hyperlipidemia rats were selected to carry out further experiments.

Anesthesia and surgery. The high-fat-treated rats were randomly divided into three groups $(n=10$ in each group; Fig. 1): A surgery group, in which rats were preloaded with $3 \mathrm{ml} / \mathrm{kg}$ saline, subsequently infused with saline at $3 \mathrm{ml} / \mathrm{kg} / \mathrm{h}$ with an osmotic pump for $2 \mathrm{~h}$, and then received anesthesia and underwent exploratory laparotomy surgery; a surgery + Dex group, in which rats received anesthesia, were preloaded with $3 \mu \mathrm{g} / \mathrm{kg}$ Dex prior to exploratory laparotomy, and subsequently received Dex infusion at $3 \mu \mathrm{g} / \mathrm{kg} / \mathrm{h}$ via an osmotic pump for $2 \mathrm{~h}$; and a sham surgery group, in which rats received anesthesia, were preloaded with $3 \mathrm{ml} / \mathrm{kg}$ saline and subsequently infused with saline at $3 \mathrm{ml} / \mathrm{kg} / \mathrm{h}$ with an osmotic pump for $2 \mathrm{~h}$. The selected DEX concentration $(3 \mu \mathrm{g} / \mathrm{kg})$ was 


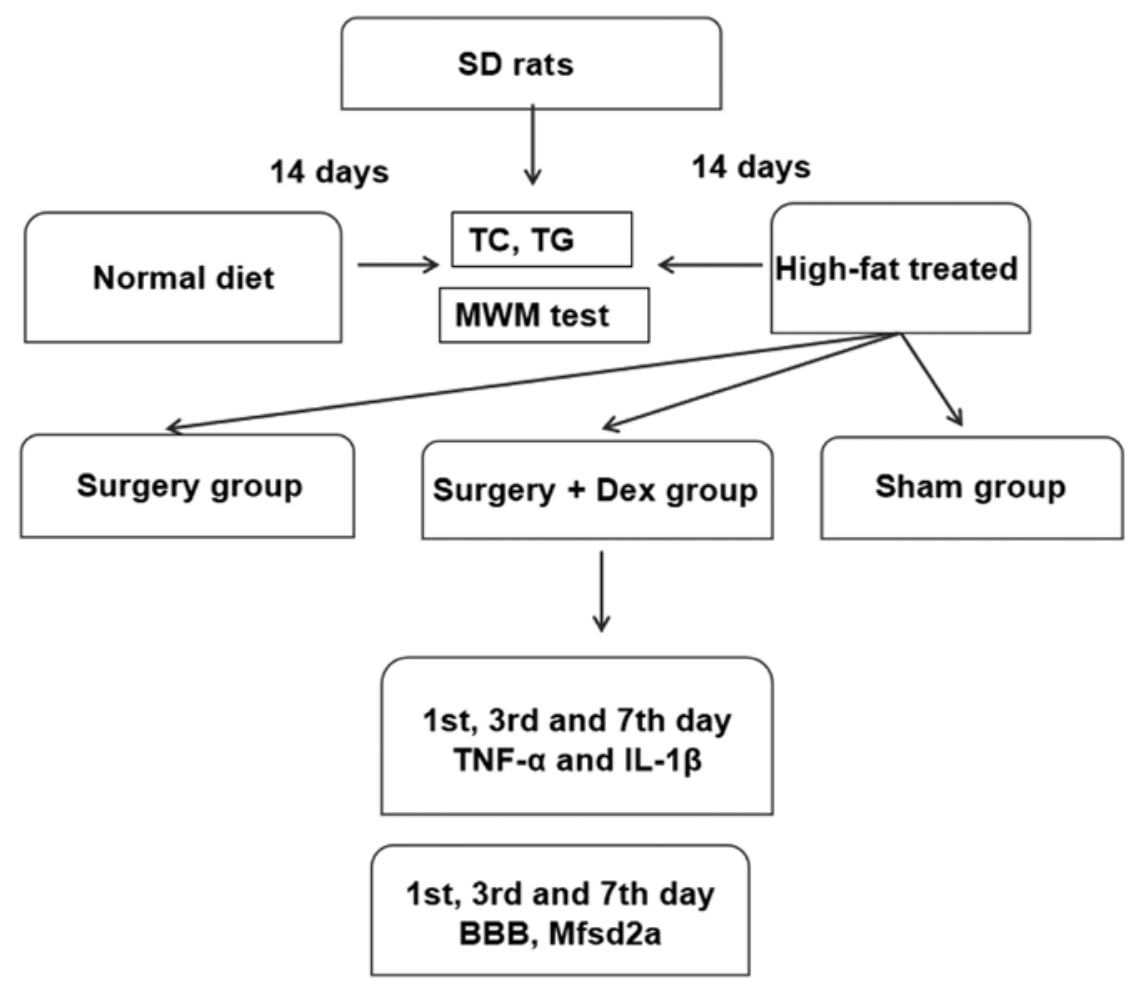

Figure 1. Experimental flow chart. There were two stages to our study. i) Establishment of the hyperlipidemia model. The two groups were fed a normal diet and a high-fat emulsion diet. These groups were established by 14 days of feeding. ii) Establishment of the surgical groups. The sham group was fed the high-fat diet and underwent the sham surgery procedure; the surgery group was fed the high-fat diet, received anesthesia, and underwent exploratory laparotomy surgery; and the surgery+Dex group was fed the high-fat diet, received anesthesia, received preloading treatment with $3 \mu \mathrm{g} / \mathrm{kg}$ Dex prior to exploratory laparotomy surgery, underwent exploratory laparotomy surgery, and then received subsequent infusion of Dex at $3 \mu \mathrm{g} / \mathrm{kg} / \mathrm{h}$ via an osmotic pump for $2 \mathrm{~h}$. BBB, blood-brain barrier; Mfsd2a, major facilitator superfamily domain-containing protein 2; TNF- $\alpha$, tumor necrosis factor- $\alpha$; IL-1 $\beta$, interleukin-1 $\beta$; TG, triglyceride; TC, total cholesterol; MWM, Morris water maze.

determined to provide the most significant protective effect in a preliminary experiment. For the POCD model, brief anesthesia ( $1.5 \%$ sevoflurane for $5 \mathrm{~min}$ ) was administered in an anesthetic chamber. Rats were endotracheal intubated and mechanically ventilated with $1-2 \%$ sevoflurane in $100 \% \mathrm{O}_{2}$. For the exploratory laparotomy, the abdominal region was shaved and sterilized, and a 3-cm vertical incision was made approximately $0.5 \mathrm{~cm}$ below the lower right rib. The gastrointestinal tract was exteriorized, and the upper mesenteric artery clamped for $30 \mathrm{~min}$, leading to restriction of blood flow in the mesenteric vascular bed. The rats in the sham surgery group were treated in the same way without laparotomy. All rats were kept under sevoflurane anesthesia for $2 \mathrm{~h}$ and given analgesia ( $0.25 \%$ bupivacaine, $0.03 \mathrm{mg} / \mathrm{kg}$ subcutaneously) at the end of surgery. Prior to abdominal surgery, the femoral vein of the rats was released from clamping to allow anesthetic drug or saline infusion. In order to alleviate incision pain for the animals, local anesthetics were used during sealing of the incision. In the event that any animal experienced death, severe infection, incision dehiscence, and weakness, they were excluded from the experiment. No experimental animals lost more than $15-20 \%$ of their body weight or were unable to eat independently for more than $24 \mathrm{~h}$.

Morris water maze (MWM). MWM testing was used to assess spatial learning, spatial memory, and cognitive abilities. The water maze consisted of a circular pool (diameter of $150 \mathrm{~cm}$, height of $50 \mathrm{~cm}$, black interior wall) that was filled with water kept at a temperature of $26 \pm 1^{\circ} \mathrm{C}$. The maze was divided into four quadrants with equidistantly placed cameras in each quadrant that were connected to a computerized image analysis system. These cameras were used to record the swimming course of the rats. A transparent cylinder platform (diameter of $10 \mathrm{~cm}$, height of $27 \mathrm{~cm}$ ) was placed in the center of a quadrant, $10 \mathrm{~cm}$ underwater. All rats were trained three times a day for 6 consecutive days. The training involved the hidden platform discovery experiment and the space exploration experiment (19), with hidden platform discovery training occurring first, followed by the space exploration training on the same day. After 6 days of training, both assessments were made on days 1,3 , and 7 after surgery.

For the hidden platform discovery experiment, rats were trained to find the hidden platform. Daily training started at 10:00 am and consisted of three sessions that lasted for $1 \mathrm{~h}$ and were repeated three times a day. Each rat was sequentially placed in each quadrant and allowed to find the platform. The order of the quadrants was randomly chosen. The experiment was concluded when the rat found the platform and remained on it for $10 \mathrm{sec}$. At the completion of the experiment, the rats were wiped dry with towels and returned to their cages. The average time to find the platforms was recorded as the space learning ability.

Space exploration experiments were conducted by removing the platform in order to record the swimming course of the rats over a 35-sec duration. At 10:00 am, a probe trial was performed to assess spatial memory. The platform was 
removed from the maze. Each rat was randomly placed in one of the quadrants and allowed to explore the maze for $60 \mathrm{sec}$. Behavior was analyzed with the computerized image analyzing system. Moving distance and time spent were measured as indices of spatial memory. Our water maze detection device has a lifting platform, thus before each test, the platform was raised to the water level, allowing the animals to see the platform. When the animals were placed in the swimming pool, if they swam directly to the platform without any difficulty, this showed that their swimming ability and vision were normal and they could be used for the experiment.

TNF- $\alpha$ and IL-1 $\beta$ measurement. Serum levels of TNF- $\alpha$ and IL- $1 \beta$ were measured using ELISA kits (Wuhan USCN Business Co., Ltd.). For the measurements, 2-3 ml blood was collected from the orbital venous plexus on days 1,3 and 7 after surgery at the end of the light phase and coagulated for $30 \mathrm{~min}$. Serum was collected via centrifugation $(1,000 \mathrm{x} \mathrm{g}$ for $10 \mathrm{~min})$. ELISAs were performed according to the manufacturer's instructions. Briefly, microtiter plates (96-well flat-bottom) were coated for $24 \mathrm{~h}$ with the samples diluted at a ratio of 1:2 in diluent to a final volume of $100 \mu \mathrm{l}$. The samples were analyzed in duplicate. The plates were washed three times with diluent, and monoclonal anti-TNF- $\alpha$ (cat. no. SEA133Ra) or anti-IL-1 $\beta$ (cat. no. SEA563Ra) antibodies from Wuhan USCN Business Co., Ltd., diluted 1:1,000 in diluent were added to each well and incubated for $3 \mathrm{~h}$ at room temperature. After washing, a peroxidase-conjugated anti-rabbit secondary antibody (diluted 1:1,000) was added to each well and incubated at room temperature for $1 \mathrm{~h}$. After addition of streptavidin-enzyme, substrate and stop solution, the concentrations of TNF- $\alpha$ and IL-1 $\beta$ were determined based on the absorbance at $450 \mathrm{~nm}$ in a spectrophotometer. The standard curve demonstrated a direct relationship between optical density (OD) and test concentrations. Total protein was measured by Lowry's method using bovine serum albumin (BSA) as a standard.

Measurement of $\mathrm{BBB}$ permeability. BBB permeability was evaluated by Evans blue (Solarbio) extravasation measurements. Rats were anesthetized with $2 \%$ sevoflurane and injected with $2 \%$ Evans blue dye intravenously $(4 \mathrm{ml} / \mathrm{kg}$ ) through the femoral vein. After $1 \mathrm{~h}$, rats were perfused with $200 \mathrm{ml}$ normal saline through the left ventricle and sacrificed for hippocampus tissue collection. Hippocampal samples were homogenized in $3 \mathrm{ml}$ cold $7.5 \%$ trichloroacetic acid (Solarbio) and centrifuged at $10,000 \times \mathrm{g}$ for $10 \mathrm{~min}$. The absorbance of each hippocampus tissue extraction was measured at $632 \mathrm{~nm}$ using a spectrophotometer (Synergy H1 Hybrid Reader; BioTek Instruments, Inc.). Evans blue dye content was expressed as $\mu \mathrm{g} / \mathrm{mg}$ of hippocampal tissue and evaluated against positive control standard curves.

Quantitative real-time PCR ( $q P C R)$ analysis for $M f s d 2 a$. Total RNA was extracted from brain tissue using the RNAiso Plus Purification Kit (9108; Takara Biotechnology Co., Ltd.). RNA was evaluated by A260 (OD) measurement and integrity was checked by $2 \%$ agarose gel electrophoresis. RNA was reverse transcribed into cDNA using PrimeScript ${ }^{\mathrm{TM}}$ RT Reagent Kit for gDNA Eraser Reverse Transcript Reagent Kit (RR047A; Takara Biotechnology Co., Ltd.). qPCR was performed in a
LightCycler machine (Roche) with commercial SYBR-Green reaction reagent (RR820A; Takara Biotechnology Co., Ltd.). GAPDH was used as an internal control. The conditions for PCR consisted of 40 cycles of $95^{\circ} \mathrm{C}$ for $5 \mathrm{sec}$ and $60^{\circ} \mathrm{C}$ for $20 \mathrm{sec}$ followed by extension at $72^{\circ} \mathrm{C}$ for $10 \mathrm{~min}$. The primer sequences used for gene amplification (Invitrogen; Thermo Fisher Scientific, Inc.) were as follows: MFSD2A-forward, 5'-CCACATTCACCATCCCTATCT-3' and reverse, 5'-TTC TTATTCTGTCGCCGCTTC-3', GAPDH-forward, 5'-ATG CCGCCTGGAAACC-3' and reverse, 5'-GCATCAAAGTGG AAGAATGG-3'. The amplified products were $199 \mathrm{bp}$ in length; gene expression was quantified via the $2^{-\Delta \Delta \mathrm{Cq}}$ method (20).

Statistical analysis. All data were analyzed with SPSS version 20 (SPSS, Inc., Chicago, IL, USA). Statistical data are expressed as mean \pm standard error of the mean (SEM). MWM results were assessed with repeated measures analysis of variance (ANOVA) with Bonferroni post-test. All other data were analyzed with one-way ANOVA with Bonferroni post-test. Effects were regarded as significantly different at $\mathrm{P}<0.05$.

\section{Results}

High-fat diet feeding induces hyperlipidemia. After 14 days of feeding on the normal diet, the serum TC concentration was $1.65 \pm 0.20 \mathrm{mmol} / \mathrm{l}$ and the serum TG concentration was $0.38 \pm 0.10 \mathrm{mmol} / 1$. Comparatively, the rats fed the high-fat diet had a serum TC concentration of $3.33 \pm 0.59 \mathrm{mmol} / 1$ and serum TG concentration of $0.63 \pm 0.18 \mathrm{mmol} / \mathrm{l}$. Thus, the serum TC and TG concentrations were elevated in the high-fat-treated rats compared with those in the rats fed a normal diet (Table I; both $\mathrm{P}<0.05$ ), indicating the development of hyperlipidemia. Hyperlipidemia was maintained by continual high-fat emulsion lavage.

Cognitive function is reduced by both high-fat diet and surgery, and Dex treatment prevents POCD. After training on the escape latency test, the rats were able to learn and remember the location of the platform (Fig. 2A and D). In the space exploration test, the time and distance ratio of the high-fat treated rats were lower for targeting the quadrant than those of the rats fed a normal diet (Fig. $2 \mathrm{~B}$ and $\mathrm{C}, \mathrm{P}<0.01$ ), indicating hyperlipidemia-induced cognitive impairment.

After surgery, the time and distance ratio of the sham and surgery+Dex rats were both higher than those of the surgery group on days 1 and 3 (Fig. $2 \mathrm{E}$ and $\mathrm{F}, \mathrm{P}<0.05$ ), with no significant difference between them. The differences between the sham and surgery groups indicate that surgical stimulation of POCD occurred in the early post-operative period, and the differences between the surgery+Dex and surgery groups suggest that Dex prevented the occurrence of POCD. On day 7 , there were no differences among the groups.

The surgery-induced inflammatory response is alleviated by Dex treatment. The serum TNF- $\alpha$ concentrations in both the sham and surgery+Dex groups were lower than that in the surgery group on day 1 (Fig. $3 \mathrm{~A}$, both $\mathrm{P}<0.01$ ), and the TNF- $\alpha$ concentration was higher in the surgery+Dex group than that noted in the sham group $(\mathrm{P}<0.01)$. On day 3 , the TNF- $\alpha$ concentrations in the sham and surgery+Dex groups 
Table I. Serum total cholesterol (TC) and triglyceride (TG) levels after high-fat treatment.

\begin{tabular}{lcccccc}
\hline & & \multicolumn{2}{c}{ Day 14} & & \multicolumn{2}{c}{ Day 20 } \\
\cline { 3 - 4 } \cline { 6 - 7 } Group & Dose & TC $(\mathrm{mmol} / \mathrm{l})$ & $\mathrm{TG}(\mathrm{mmol} / \mathrm{l})$ & & $\mathrm{TC}(\mathrm{mmol} / \mathrm{l})$ & $\mathrm{TG}(\mathrm{mmol} / \mathrm{l})$ \\
\hline Saline & $10 \mathrm{ml} / \mathrm{kg}$ & $1.65 \pm 0.20$ & $0.38 \pm 0.10$ & & $1.53 \pm 0.20$ & $0.30 \pm 0.10$ \\
High-fat treated & $10 \mathrm{ml} / \mathrm{kg}$ & $3.33 \pm 0.59^{\mathrm{b}}$ & $0.63 \pm 0.18^{\mathrm{a}}$ & & $4.28 \pm 0.59^{\mathrm{b}}$ & $0.72 \pm 0.18^{\mathrm{a}}$ \\
\hline
\end{tabular}

${ }^{\mathrm{a}} \mathrm{P}<0.05$ and ${ }^{\mathrm{b}} \mathrm{P}<0.01$ compared to saline-treated rats fed a normal diet.
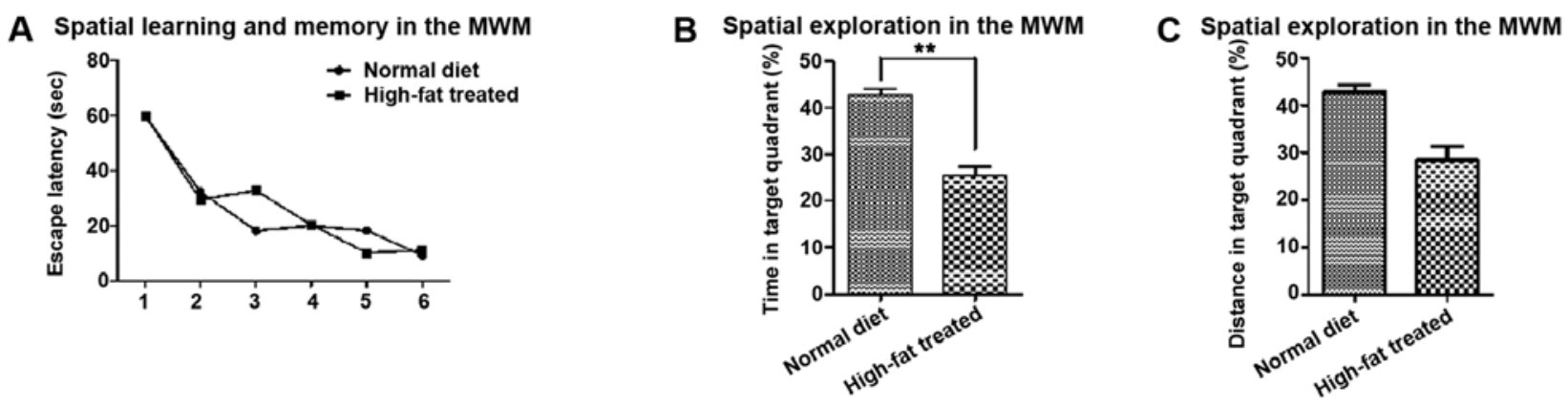

Spatial learning and memory in the MWM

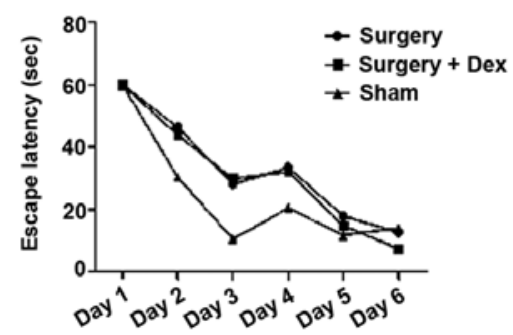

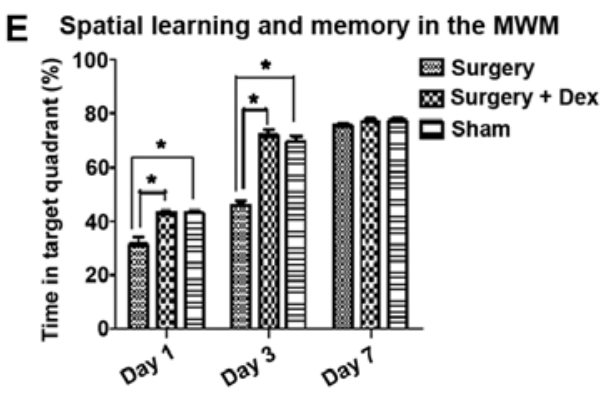

Figure 2. Learning memory and exploration performance following surgery. (A) Spatial learning and memory in the MWM. Escape latency (sec) was recorded for the six training sessions. (B) Spatial learning and memory in the MWM. Escape latency (sec) was recorded for the six training sessions. (C) Spatial exploration in the MWM. Time in target quadrant. (D) Spatial exploration in the MWM. Distance in target quadrant. ${ }^{* *} \mathrm{P}<0.01$ and ${ }^{*} \mathrm{P}<0.05$. There were significant effects of training on escape latency test (A and D). In the space exploration test, the time and distance ratio of the high-fat-treated rats were lower than those of the rats fed a normal diet in targeting the quadrant ( $\mathrm{B}$ and $\mathrm{C}, \mathrm{P}<0.01$ ). In addition, the time and distance ratios of the sham group were higher than those of the surgery group. The time and distance ratio of the surgery+Dex group were also higher than those of the surgery group on days 1 and 3 (E and F, P<0.05). On day 7 , there were no differences among the groups. MWM, Morris water maze.

were still lower than that in the surgery group (Fig. 3A, both $\mathrm{P}<0.05)$, but no difference was observed in the TNF- $\alpha$ concentrations in the sham and surgery+Dex groups. These results revealed that anesthesia and exploratory laparotomy induced an inflammatory response and that Dex treatment alleviated this inflammatory response in the early post-operative period. On day 7 post-operatively, no significant differences in serum TNF- $\alpha$ concentration were observed among the groups, indicating that the inflammation induced by surgery had returned to baseline levels.

The serum IL-1 $\beta$ concentrations in the surgery and surgery+Dex groups were both higher than that in the sham group on day 1 (Fig. 3B, $\mathrm{P}<0.01$ ). On day 3 however, while the IL-1 $\beta$ concentration in the surgery group was still higher than that in the sham group $(\mathrm{P}<0.05)$, the IL-1 $\beta$ concentration in the surgery+Dex group had decreased to less than that in the surgery group $(\mathrm{P}<0.05)$, implying a reduction in inflammation with Dex treatment.
$B B B$ permeability. Quantification of the observed Evans Blue staining in each group revealed significantly more staining in the surgery group than in the sham group on day 1 (Fig. 4 , $\mathrm{P}<0.01$ ), indicating that surgery induced BBB dysfunction. In addition, Evans Blue staining was significantly reduced in the surgery+Dex compared with the surgery group $(\mathrm{P}<0.01)$ and not statistically different from that in the sham group on day 1 ( $\mathrm{P}>0.05)$, indicating that Dex treatment attenuated the increase in BBB permeability caused by surgical trauma. No differences were observed among the groups on days 3 and 7.

Downregulation of $M f s d 2$ a mRNA expression in the early postoperative period is prevented by Dex treatment. The relative expression of $M f s d 2 a$ mRNA in the surgery group was significantly lower than that in the sham group on days 1 and 3 (Fig. 5, both $\mathrm{P}<0.05$ ), indicating that $M f s d 2 a$ mRNA expression was downregulated in the early post-operative period. 

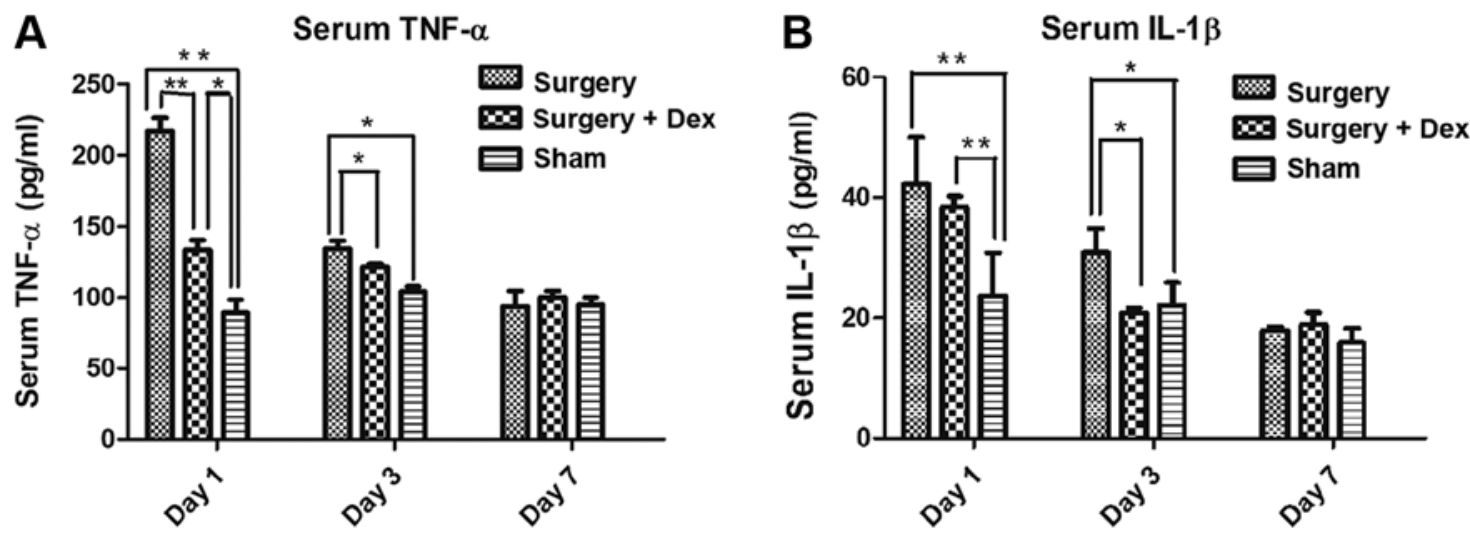

Figure 3. Serum inflammatory factor concentrations following surgery. (A) Serum TNF- $\alpha$ concentrations (pg/ml). (B) Serum IL-1 $\beta$ concentrations (pg/ml). ${ }^{* *} \mathrm{P}<0.01,{ }^{*} \mathrm{P}<0.05$. The TNF- $\alpha$ concentrations in the sham group and surgery+Dex group were lower than that in the surgery group on days 1 and 3 (A, both $\mathrm{P}<0.01)$. On day 7 , there were no significant differences among groups. The serum IL-1 $\beta$ concentrations in the surgery group and surgery+Dex group were both higher than that in sham group on day $1(\mathrm{~B}, \mathrm{P}<0.01)$. However, on day 3 , while the IL-1 $\beta$ concentration in the surgery group was still higher than that in the sham group $(\mathrm{P}<0.05)$, the concentration in the surgery+Dex group had decreased to less than that in the surgery group $(\mathrm{P}<0.05)$. TNF- $\alpha$, tumor necrosis factor- $\alpha$; IL- $1 \beta$, interleukin- $1 \beta$.

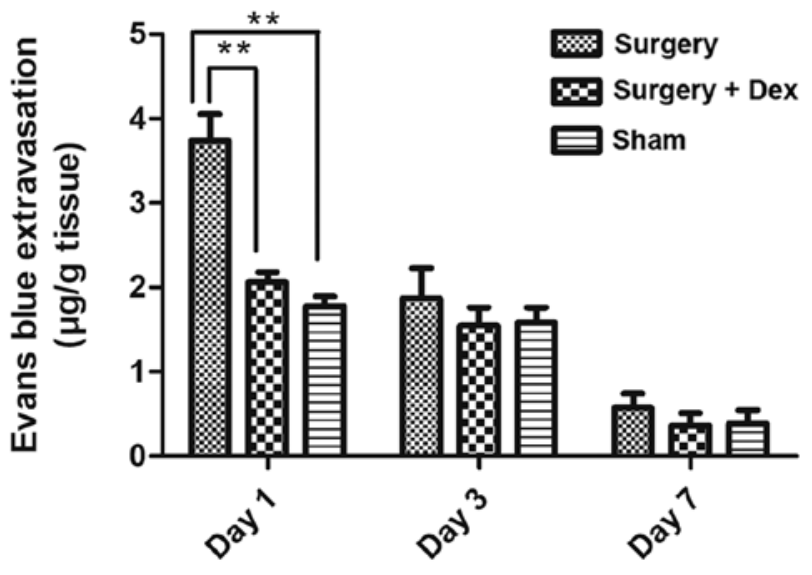

Figure 4. BBB permeability in the hippocampus was determined by Evans Blue staining and quantified on days 1,3 , and 7 following surgery. ${ }^{* * *} \mathrm{P}<0.01$ compared to the surgery group. Quantified Evans Blue staining was higher in the surgery group than that in the sham group on day $1(\mathrm{P}<0.01)$ and significantly lower in the surgery+Dex group compared with the surgery group on day $1(\mathrm{P}<0.01)$. BBB, blood-brain barrier.

However, $M f s d 2 a$ expression did not differ between the sham and surgery+Dex groups on days 1 and 3 , with significantly higher $M f s d 2 a$ expression observed in the surgery+Dex group compared with the surgery group at these time points $(\mathrm{P}<0.05)$. These results indicate that the downregulation of $M f s d 2 a$ following surgery was prevented by Dex treatment. By day 7 , no differences in relative expression of $M f s d 2 a$ mRNA were observed among the three groups.

\section{Discussion}

The aim of the present study was to evaluate the effect of dexmedetomidine (Dex) on the incidence of postoperative cognitive dysfunction (POCD) in an animal model of hyperlipidemia-induced blood-brain barrier (BBB) dysfunction, through its effects on $M f s d 2 a$ gene expression. The major findings of the present study were that: i) Anesthesia and surgery increased BBB permeability and, correspondingly,

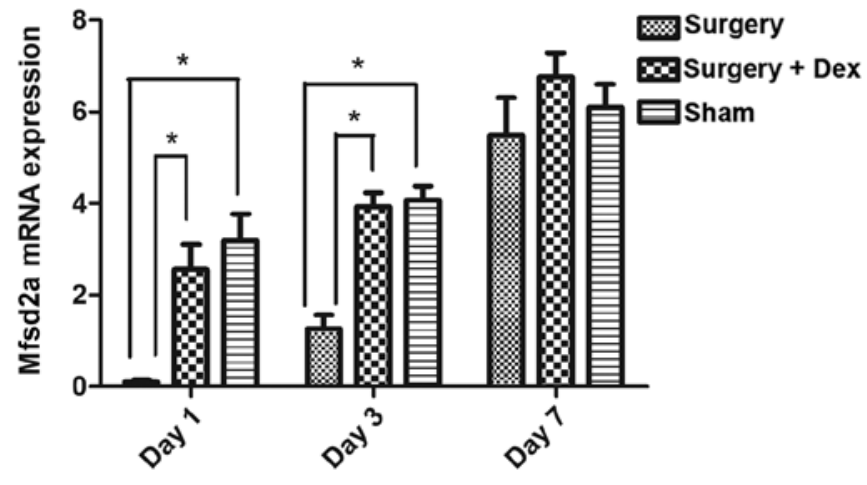

Figure 5. $M f s d 2 a$ mRNA expression measured by qPCR analysis. $M f s d 2 a$ expression was lower in the surgery group than in the sham group and surgery+Dex group on days 1 and $3(\mathrm{P}<0.05)$. On day 7 , no differences were observed among the groups. ${ }^{*} \mathrm{P}<0.05$ compared to the sham group. Mfsd2a, major facilitator superfamily domain-containing protein 2; qPCR, quantitative real-time PCR.

induced cognitive dysfunction, and ii) treatment with Dex attenuated these effects. Combined, our results revealed an inflammation-based mechanism for the development of POCD.

Surgery and anesthesia have been shown to induce tissue damage and activate the peripheral innate immune system, resulting in the release of inflammatory mediators (21).Following surgery, elderly patients often develop POCD, which can both lengthen the recovery process and accelerate future progression to Alzheimer's disease. Anesthetics, particularly volatile anesthetics, have been strongly associated with the development of POCD (22-24). With respect to pre-clinical studies, both cell culture and animal studies have suggested that anesthetics may stimulate neuroapoptosis, caspase activation, $\beta$-amyloid protein (A $\beta$ ) accumulation and oligomerization, and, ultimately, neurodegeneration and deficits in neurocognition. While the primary risk factors for POCD are increased age, surgery, and use of anesthesia, other risk factors include hyperlipidemia, diabetes mellitus, obesity, vascular factors, and depression, all of which play a role in the pathogenesis of POCD (25). Among these risk factors, the effects of hyperlipidemia have been largely understudied. 
Hyperlipidemia is a systemic disorder of lipid metabolism resulting in elevated levels of fats in the blood, including total cholesterol (TC), triglycerides (TG), free fatty acids, high-density lipoprotein (HDL), very low-density lipoprotein (VLDL), and low-density lipoprotein (LDL), which may eventually result in atherosclerosis. Atherosclerosis is a known trigger for immune responses that drives neuroinflammation and neurodegeneration. Evaluation of the effects of anesthetics on cognitive dysfunction in hyperlipidemia animal models have not, to date, been reported. In our experiments, rats fed a high-fat emulsion for 14 days developed elevated serum TC and TG levels, which led to learning and memory disturbances according to results obtained using the Morris water maze. The Morris water maze is a particularly useful tool for judging the spatial learning and memory ability of mice (26). It can provide more experimental parameters for systematically and comprehensively investigating the spatial cognitive processing of experimental animals and objectively reflects their cognitive level. The learning and memory impairment and sensory and motor impairment of experimental animals were separated to reduce their interference in the detection of learning and memory processing. Additionally, our laboratory experience with using the Morris water maze to detect behavioral changes in animals is superior to that for other experimental methods.

The BBB limits the entry of plasma components, red blood cells, and leukocytes into the brain (27). The BBB is often disrupted after ischemic injury, inflammation, or in a variety of vascular disorders that can generate neurotoxic products, thereby compromising synaptic and neuronal functions. Furthermore, an intact BBB is a major obstacle for drug delivery to the central nervous system (CNS). A limited understanding of the molecular mechanisms that control BBB formation has hindered our ability to manipulate the BBB in disease and drug therapy. Therefore, understanding how the BBB is impaired following surgery could provide insight and aid in improving therapies targeting the CNS (28). Major facilitator superfamily domain-containing protein 2 (Mfsd2a) is a key regulator of BBB function that may act by suppressing transcytosis in CNS-specific endothelial cells. Mfsd2a, therefore, may play a protective role in maintaining BBB function (29). In the present study, $M f s d 2 a$ expression was reduced following surgery, an effect that was inhibited by Dex treatment. While this effect on $M f s d 2 a$ expression provides insight into the mechanism of the therapeutic effects of Dex, future studies of additional genes will be valuable. In a rat model of lipopolysaccharide (LPS)-induced neuroinflammation, additional genes showed altered expression after Dex treatment (30). These include the IL1- $\beta$ and TNF- $\alpha$ genes as well as miR132 expression and deacetylation of histone H3. Dex decreased the LPS-induced reductions in IL1- $\beta$ and TNF- $\alpha$ gene expression levels and reversed the A $\beta$-induced deacetylation of histone $\mathrm{H} 3$, thereby, increasing BDNF production. Studies of the effects of their altered expression following Dex treatment are warranted. A previous study showed that treatment with Dex reduced inflammation after trauma, increased the expression of tight junction proteins, and diminished secondary BBB damage and apoptosis, and these protective effects were likely mediated via reduced activation of the nuclear factor (NF)- $\mathrm{KB}$ and NLRP3 inflammasome pathways (31). Future research into the various effects of Dex treatment will provide a more comprehensive understanding of how the drug can be used clinically.

The neuroprotective properties of Dex are largely attributed to its agonistic actions on $\alpha 2$-adrenoreceptors, which belong to the G-protein coupled family of transmembrane receptors and are present on both pre- and post-synaptic autonomic ganglia in the central and peripheral nervous systems (15). Binding of agonists, whether endogenous (norepinephrine) or exogenous (dexmedetomidine), results in G-protein coupling with the inhibition of both adenylyl cyclase and phospholipase $\mathrm{C}$ activity and additional downstream effects caused by such inhibition. Neuroinflammation may be the principal cause for cognitive dysfunction after general anesthesia or surgery in rodents. The results of the present study demonstrated that Dex improved learning and memory function in the early postoperative period in a rat model of hyperlipidemia.

Surgical trauma and anesthesia activate the immune response of both the peripheral and CNS. Surgical trauma will inevitably affect the blood pressure and respiration of animals, leading to changes in the synthesis and release of inflammatory factors. Therefore, in the pre-experimental stage, we performed one femoral artery puncture manometry and another femoral vein administration and blood collection in rats. We found no significant effect of anesthesia and operation on blood pressure and heart rate among the groups in the same experiment. Therefore, femoral artery puncture was not performed in order to reduce the incidence of infection after operation in the water maze experiment.

An increase in inflammatory cytokines in the serum may be an important risk factor for cognitive decline after surgery (32). We did not include a group treated with Dex after sham surgery, as surgical trauma is the inducing factor for POCD in rats. A surgery group, in which rats received anesthesia and surgery served as a positive control group. The impairment of surgical trauma on cognitive function of rats in the sham-operation group was slightly less than that in operation group, which was regarded as a negative control group. The Dex group was used as the experimental group to observe the protective effect of Dex on cognitive function. Thus, Dex treatment without surgery would not provide useful information for the purpose of our study.

Both TNF- $\alpha$ and IL-1 $\beta$ have been implicated in the development of cognitive dysfunction (33). Pro-inflammatory cytokines can inhibit long-term potentiation and the expression of brain-derived neurotrophic factor in the hippocampus. Moreover, TNF- $\alpha$ and IL-1 $\beta$ are known to contribute to neuroinflammation in many CNS disorders, as well as the development of inflammatory responses within the brain. Dex administration has been shown to decrease the production of inflammatory cytokines in severely septic patients (15). In the present study, Dex treatment attenuated the postoperative increase in serum TNF- $\alpha$ but not IL-1 $\beta$ on days 1 and 3 after surgery. A limitation of the present study is that we measured TNF- $\alpha$ and IL-1 $\beta$ levels in serum only, and the effects of DEX on TNF- $\alpha$ and IL- $1 \beta$ concentrations in the brain should be determined in future studies.

In conclusion, our results revealed that hyperlipidemia in rats increased postoperative cognitive impairment following surgery, and these effects could be attenuated by Dex treatment. Neuronal dysfunction was ameliorated by inhibiting 
the inflammatory response and increasing $M f s d 2 a$ gene expression to preserve the integrity of the BBB. Our findings provide rationale for examining Dex as a preemptive therapy for preventing POCD in elderly patients.

\section{Acknowledgements}

The authors would like to thank Professor Shen Lv of the Research Center of the Second Affiliated Hospital of Dalian Medical University, and Miss Yu-Hua Gao of the Department of Anesthesiology, Henan Province Hospital of Traditional Chinese Medicine, for their technical support.

\section{Funding}

This research was supported by funding from the Science and Technology Department of Liaoning Province of China (grant no. 2013023009). The authors declare that they have no financial relationship with the organization that sponsored the research, and the funding body was not involved in study design, data collection, analysis and writing of the study.

\section{Availability of data and materials}

The datasets used and/or analyzed during the current study are available from the corresponding author on reasonable request.

\section{Authors' contributions}

XPZ and DBL conceived and designed the experiments. XPZ and YRL performed the experiments. YRL analyzed the data. XPZ wrote the manuscript. MC conducted data analysis and edited the manuscript. HTY was involved in performing the animal behavioral tests. GW served an important role in the establishment of the hyperlipidemia animal model. MH determined the dose of Dex used in the experiments. DBL provided guidance during the whole process and assisted writing the article, and ensured the integrity of experiments and approved the final version. All authors edited and approved the submission and agree to be accountable for all aspects of the research in ensuring that the accuracy or integrity of any part of the work are appropriately investigated and resolved. DBL is the corresponding author.

\section{Ethics approval and consent to participate}

All animal procedures were approved by the Animal Experiment Ethics Committee of Dalian Medical University, China. All studies involving animals are reported in accordance with the ARRIVE guidelines for reporting experiments involving animals.

\section{Patient consent for publication}

Not applicable.

\section{Competing interests}

The authors declare that they have no competing interests.

\section{References}

1. Pappa M, Theodosiadis N, Tsounis A and Sarafis P: Pathogenesis and treatment of post-operative cognitive dysfunction. Electron Physician 9: 3768-3775, 2017

2. Bilotta F, Qeva E and Matot I: Anesthesia and cognitive disorders: A systematic review of the clinical evidence. Expert Rev Neurother 16: 1311-1320, 2016.

3. Price CC, Garvan CW and Monk TG: Type and severity of cognitive decline in older adults after noncardiac surgery. Anesthesiology 108: 8-17, 2008.

4. Lin Y, Chen J and Wang Z: Meta-analysis of factors which influence delirium following cardiac surgery. J Card Surg 27: 481-492, 2012.

5. Loffler T, Flunkert S, Temmel M and Hutter-Paier B: Decreased plasma Abeta in hyperlipidemic APPSL transgenic mice is associated with BBB dysfunction. Front Neurosci 10: 232, 2016.

6. Ullrich C, Pirchl M and Humpel C: Hypercholesterolemia in rats impairs the cholinergic system and leads to memory deficits. Mol Cell Neurosci 45: 408-417, 2010.

7. Cunha S, Amaral MH, Lobo JM and Silva AC: Therapeutic strategies for Alzheimer's and Parkinson's diseases by means of drug delivery systems. Curr Med Chem 23: 3618-3631, 2016.

8. Acharya NK, Goldwaser EL, Forsberg MM, Godsey GA Johnson CA, Sarkar A, DeMarshall C, Kosciuk MC, Dash JM, Hale CP, et al: Sevoflurane and Isoflurane induce structural changes in brain vascular endothelial cells and increase blood-brain barrier permeability: Possible link to postoperative delirium and cognitive decline. Brain Res 1620: 29-41, 2015.

9. Ben-Zvi A, Lacoste B, Kur E, Andreone BJ, Mayshar Y, Yan H and $\mathrm{Gu} \mathrm{C}$ : Mfsd2a is critical for the formation and function of the blood-brain barrier. Nature 509: 507-511, 2014.

10. Berger JH, Charron MJ and Silver DL: Major facilitator superfamily domain-containing protein $2 \mathrm{a}$ (MFSD2A) has roles in body growth, motor function, and lipid metabolism. PLoS One 7: e50629, 2012.

11. Guerriero F, Sgarlata C, Francis M, Maurizi N, Faragli A, Perna S, Rondanelli M, Rollone M and Ricevuti G: Neuroinflammation, immune system and Alzheimer disease: Searching for the missing link. Aging Clin Exp Res 29: 821-831, 2016.

12. Barrientos RM, Hein AM, Frank MG, Watkins LR and Maier SF: Intracisternal interleukin-1 receptor antagonist prevents postoperative cognitive decline and neuroinflammatory response in aged rats. J Neurosci 32: 14641-14648, 2012.

13. Jolkkonen J, Puurunen K, Koistinaho J, Kauppinen R, Haapalinna A, Nieminen L and Sivenius J: Neuroprotection by the alpha2-adrenoceptor agonist, dexmedetomidine, in rat focal cerebral ischemia. Eur J Pharmacol 372: 31-36, 1999.

14. Mantz J, Josserand J and Hamada S: Dexmedetomidine: new insights. Eur J Anaesthesiol 28: 3-6, 2011.

15. Venn RM, Bryant A, Hall GM and Grounds RM: Effects of dexmedetomidine on adrenocortical function, and the cardiovascular, endocrine and inflammatory responses in post-operative patients needing sedation in the intensive care unit. Br J Anaesth 86: 650-656, 2001.

16. Goyagi T: Dexmedetomidine reduced sevoflurane-induced neurodegeneration and long-term memory deficits in neonatal rats. Int J Dev Neurosci 75: 19-26, 2019.

17. Akpinar H, Naziroglu M, Ovey IS, Cig B and Akpinar O: The neuroprotective action of dexmedetomidine on apoptosis, calcium entry and oxidative stress in cerebral ischemia-induced rats: Contribution of TRPM2 and TRPV1 channels. Sci Rep 6: 37196, 2016.

18. Zhang C, Li J, Wang J, Song X, Zhang J, Wu S, Hu C, Gong Z and Jia L: Antihyperlipidaemic and hepatoprotective activities of acidic and enzymatic hydrolysis exopolysaccharides from Pleurotus eryngii SI-04. BMC Complement Altern Med 17: 403, 2017.

19. Tomas Pereira I and Burwell RD: Using the spatial learning index to evaluate performance on the water maze. Behav Neurosci 129: 533-539, 2015.

20. Livak KJ and Schmittgen TD: Analysis of relative gene expression data using real-time quantitative PCR and the 2(-Delta Delta C(T)) method. Methods 25: 402-408, 2001.

21. Hovens IB, Schoemaker RG, van der Zee EA, Absalom AR, Heineman E and van Leeuwen BL: Postoperative cognitive dysfunction: Involvement of neuroinflammation and neuronal functioning. Brain Behav Immun 38: 202-210, 2014.

22. Hu N, Wang C, Zheng Y, Ao J, Zhang C, Xie K, Li Y, Wang H, Yu Y and Wang G: The role of the Wnt/ $\beta$-catenin-Annexin A1 pathway in the process of sevoflurane-induced cognitive dysfunction. J Neurochem 137: 240-252, 2016. 
23. Vlisides $\mathrm{P}$ and Xie Z: Neurotoxicity of general anesthetics: an update. Curr Pharm Des 18: 6232-6240, 2012.

24. Bilotta F, Gelb AW, Stazi E, Titi L, Paoloni FP and Rosa G: Pharmacological perioperative brain neuroprotection: A qualitative review of randomized clinical trials. Br J Anaesth 110 (Suppl 1): i113-i120, 2013.

25. Di Cataldo V, Geloen A, Langlois JB, Chauveau F, Theze B, Hubert V, Wiart M, Chirico EN, Rieusset J, Vidal H, et al: Exercise does not protect against peripheral and central effects of a high cholesterol diet given ad libitum in old $\mathrm{ApoE}^{-/-}$mice. Front Physiol 7: 453, 2016.

26. Vorhees CV and Williams MT: Morris water maze: Procedures for assessing spatial and related forms of learning and memory. Nat Protoc 1: 848-858, 2006.

27. Varatharaj A and Galea I: The blood-brain barrier in systemic inflammation. Brain Behav Immun 60: 1-12, 2017.

28. Elwood E, Lim Z, Naveed H and Galea I: The effect of systemic inflammation on human brain barrier function. Brain Behav Immun 62: 35-40, 2017.

29. Zhao $Z$ and Zlokovic BV: Blood-brain barrier: A dual life of MFSD2A? Neuron 82: 728-730, 2014.
30. Paeschke N, von Haefen C, Endesfelder S, Sifringer M and Spies CD: Dexmedetomidine prevents lipopolysaccharide-induced microRNA expression in the adult rat brain. Int J Mol Sci 18: E1830, 2017.

31. Wang D, Xu X, Wu YG, Lyu L, Zhou ZW and Zhang JN: Dexmedetomidine attenuates traumatic brain injury: Action pathway and mechanisms. Neural Regen Res 13: 819-826, 2018.

32. Tanabe K, Matsushima-Nishiwaki R, Kozawa O and Iida H: Dexmedetomidine suppresses interleukin-1beta-induced interleukin-6 synthesis in rat glial cells. Int J Mol Med 34: 1032-1038, 2014.

33. Terrando N, Monaco C, Ma D, Foxwell BM, Feldmann M and Maze M: Tumor necrosis factor-alpha triggers a cytokine cascade yielding postoperative cognitive decline. Proc Natl Acad Sci USA 107: 20518-20522, 2010.

(i)@ $\Theta$ This work is licensed under a Creative Common EY NO NO Attribution-NonCommercial-NoDerivatives 4.0 International (CC BY-NC-ND 4.0) License. 